\title{
ON THE STABILIZATION OF INTERNALLY COUPLED MAP LATTICE SYSTEMS
}

WEIHONG HUANG

Received 24 September 2003

The adaptive adjustment mechanism is applied to the stabilization of an internally coupled map lattice system defined by $x_{i, t+1}=G\left(\left(1-\alpha_{i}-\beta_{i}\right) x_{i, t}+\alpha_{i} x_{i+1, t}+\beta_{i} x_{i-1, t}\right)$, where $f: \mathbb{R} \rightarrow \mathbb{R}$ is a nonlinear map, and $\alpha$ and $\beta$ are nonnegative coupling constants that satisfy the constraint $\alpha_{i}+\beta_{i}<1$, for all $x \in \mathbb{R}, i=1,2, \ldots, n$. Sufficient conditions and ranges of adjustment parameters that guarantee the local stability of a generic steady state have been provided. Numerical simulations have demonstrated the effectiveness and efficiency for this mechanism to stabilize the system to a generic unstable steady state or a periodic orbit.

\section{Introduction}

The issue of controlling spatiotemporal chaos has attracted more and more attentions from physicists due to its broad applications in plasma, laser devices, turbulence, and chemical and biological systems. Recent advances include constant pinnings proposed by Parekh et al. [6], feedback pinnings by $\mathrm{Hu}$ and $\mathrm{Qu}$ [2], phase space compression technique by $\mathrm{Xu}$ and Shen [9], the linear control basing on symmetry property by Grigoriev and Cross [1], delayed-feedback strategies by Parmananda et al. [7], and the adaptive control proposed by Sinha and Gupte [8]. Due to the presence of numerous more unstable spatial modes resulting from spatial interactions, the control of spatiotemporal chaos, leading up to the control of turbulence, turns out to be much more complicated than the similar practice on a one-dimensional discrete system. However, most methods proposed so far are system-specific or deficient in providing convincing theoretical foundations. In [5], the adaptive adjustment mechanism [3,4] is applied to stabilize a most general type of externally coupled map lattice system. Simulations conducted have shown that such a stabilization turns out to be very effective and efficient. Stabilization of an original unstable coupled map system is usually achieved soon after the adaptive adjustment is triggered.

In the present paper, the study is extended to a new type of spatiotemporal chaos model-internally coupled map lattice system. Sufficient conditions and ranges of 
adjustment parameters are provided. Numerical simulations show the effectiveness and efficiency for this mechanism to stabilize the system to a generic unstable fixed point or a periodic orbit.

\section{Control chaos through adaptive adjustment}

Consider an $n$-dimensional dynamical process defined by

$$
\mathbf{X}_{t+1}=\mathbf{F}\left(\mathbf{X}_{t}\right),
$$

where $\mathbf{X}_{t}=\left(x_{1 t}, x_{2 t}, \ldots, x_{n t}\right)$ and $\mathbf{F}=\left(f_{1}, f_{2}, \ldots, f_{n}\right)$, with $f_{i}$ being well-defined functions on a domain in $\mathbb{R}^{n}$.

Definition 2.1. An adaptive adjustment mechanism is defined by the following adjusted process:

$$
\mathbf{X}_{t+1}=\tilde{\mathbf{F}}_{\Gamma}=(\mathbf{I}-\mathbf{\Gamma}) \mathbf{F}\left(\mathbf{X}_{\mathbf{t}}\right)+\mathbf{\Gamma} \mathbf{X}_{\mathbf{t}}
$$

where $\Gamma=\operatorname{diag}\left\{\gamma_{1}, \gamma_{2}, \ldots, \gamma_{n}\right\}$ is a diagonal matrix, with $\gamma_{i} \geq 0$, for $i=1,2, \ldots, n$, and is referred to as an adaptive parameter matrix.

A simple but effective type of adaptive adjustment is the so-called uniformly adaptive adjustment defined by

$$
\Gamma=\operatorname{diag}\{\gamma, \gamma, \ldots, \gamma\}=\gamma \mathbf{I}_{n},
$$

where $\mathbf{I}_{n}$ is an order $n$ identity matrix. That is, all state variables are adjusted with same speed:

$$
\mathbf{X}_{t+1}=\tilde{\mathbf{F}}_{\Gamma}=(1-\gamma \mathbf{F})\left(\mathbf{X}_{\mathbf{t}}\right)+\gamma \mathbf{X}_{\mathbf{t}} .
$$

Let $\overline{\mathbf{X}}$ be a fixed point of (2.1), that is, $\overline{\mathbf{X}}=\mathbf{F}(\overline{\mathbf{X}})$. Then the process $\tilde{\mathbf{F}}_{\Gamma}\left(X_{t}\right)$ shares exactly the same set of fixed points of $\overline{\mathbf{X}}$, that is, $\overline{\mathbf{X}}=\tilde{\mathbf{F}}_{\Gamma}(\overline{\mathbf{X}})$.

Let $J(\overline{\mathbf{X}})$ be the Jacobian matrix of the original process $\mathbf{F}$, evaluated at $\overline{\mathbf{X}}$, with $\left\{\lambda_{1}\right.$, $\left.\lambda_{2}, \ldots, \lambda_{n}\right\}$ being the $n$ eigenvalues solved from the following characteristic equation:

$$
\left|\lambda \mathbf{I}_{n}-\mathscr{\Phi}(\overline{\mathbf{X}})\right|=\prod_{j=1}^{n}\left(\lambda-\lambda_{j}\right)=0 .
$$

Denote a pair of complex conjugates $\lambda_{j}$ and $\bar{\lambda}_{j}$ by

$$
\lambda_{j}=a_{j}+b_{j} \mathbf{i}, \quad \bar{\lambda}_{j}=a_{j}-b_{j} \mathbf{i},
$$

with the modulus $\left|\lambda_{j}\right|=\left|\bar{\lambda}_{j}\right|=\sqrt{a_{j}^{2}+b_{j}^{2}}$. Then an unstable fixed point can be classified according to the modulus of related eigenvalues as follows. 
Definition 2.2 (classification of unstable fixed points). (i) Type I unstable fixed points: $a_{j}<1$, for all $j$, that is, the fixed points with all eigenvalues less than unity in real parts.

(ii) Type II unstable fixed points: $a_{j}>1$, for all $j$, that is, the fixed points with all eigenvalues greater than unity in real parts.

(iii) Type III unstable fixed points: $a_{i}>1, a_{j}<1$, for some $i$ and $j$, that is, the fixed points with some real parts greater than unity, others less than unity in real parts.

(iv) Type IV unstable fixed points: there exists at least one $j$ such that either $a_{j}=1$ or $\lambda_{j}=1$, that is, the fixed points with unity eigenvalues.

Then the following theorem is proved by the author in [4].

Theorem 2.3. For an $n$-dimensional dynamical process $\mathbf{X}_{t+1}=\mathbf{F}\left(\mathbf{X}_{t}\right)$, an unstable fixed point $\overline{\mathbf{X}}$ can be stabilized through uniformly adaptive adjustment defined by (2.4) if and only if $\overline{\mathbf{X}}$ is either a type I fixed point $\left(a_{j}<1\right.$ for all $\left.j=1,2, \ldots, n\right)$ or a type II fixed point $\left(a_{j}>1\right.$ for all $\left.j=1,2, \ldots, n\right)$.

On the other hand, when an unstable fixed point $\overline{\mathbf{X}}$ is of type III, an implementation of uniformly adaptive adjustment (2.4) usually leads the adjusted system to a periodic cycle. If our goal is to stabilize the system to its generic fixed point, then a nonuniformly adaptive adjustment mechanism defined by (2.2) has to be resorted. The detail discussion is offered in [4].

\section{Internally coupled map lattice systems}

Definition 3.1. A nonlinear process $\mathbf{F}(\mathbf{X})=\left\{f_{1}(\mathbf{X}), f_{2}(\mathbf{X}), \ldots, f_{n}(\mathbf{X})\right\}$, with $\mathbf{X}=\left(x_{1}, x_{2}, \ldots\right.$, $\left.x_{n}\right)$, is an internally coupled map lattice if

$$
x_{i, t+1}=f_{i}\left(\mathbf{X}_{\mathbf{t}}\right)=G\left(\left(1-\alpha_{i}-\beta_{i}\right) x_{i, t}+\alpha_{i} x_{i+1, t}+\beta_{i} x_{i-1, t}\right), \quad i=1,2, \ldots, n,
$$

with periodic boundary conditions, where $G: \mathbb{R} \rightarrow \mathbb{R}$ is a nonlinear map and $\alpha_{i} \geq 0, \beta_{i} \geq 0$ for all $i$. For convenience, we will refer to $G$ as a coupling map and to $\alpha_{i}$ and $\beta_{i}$ as coupling constants.

Definition 3.2. An internally coupled map lattice system defined by (3.1) is said to be uniformly coupled if $\alpha_{i}=\alpha$ and $\beta_{i}=\beta$ for all $i=1,2, \ldots, n$.

Definition 3.3. An internally coupled map lattice system defined by (3.1) will be referred to as backward-coupled system if $\alpha_{i}=0$, for all $i$, and as forward-coupled system if $\beta_{i}=0$, for all $i$.

If $\bar{x}$ is a fixed point of one-dimensional map, that is, $G(\bar{x})=\bar{x}$, then $\mathbf{X}^{-}=(\bar{x}, \bar{x}, \ldots, \bar{x})$ is also a fixed point of $\mathbf{F}$. For the convenience of reference, we will refer to $\mathbf{X}^{-}=(\bar{x}, \bar{x}, \ldots, \bar{x})$ as a generic fixed point of (3.1).

Then we have the following lemma.

Lemma 3.4. A generic fixed point $\overline{\mathbf{X}}=(\bar{x}, \bar{x}, \ldots, \bar{x})$ of an internally coupled map lattice system defined by (3.1) is unstable if $\bar{x}$ is an unstable fixed point of $G$.

Moreover, if $\epsilon_{i}=\alpha_{i}+\beta_{i} \leq 1$ for all $i$, then $\bar{X}$ is stable if and only if $\bar{x}$ is stable. 
Proof. Denote $\sigma=G^{\prime}(\bar{x})$. The Jacobian matrix of $\mathbf{F}$ evaluated at $\overline{\mathbf{X}}$, denoted by $\mathscr{E}=$ $\left[j_{i j}\right]_{n \times n}$, can be expressed as

$$
\mathscr{J}=\left[\begin{array}{ccccc}
\left(1-\alpha_{1}-\beta_{1}\right) \sigma, & \alpha_{1} \sigma, & 0, & \ldots & \beta_{1} \sigma \\
\vdots & \ddots & \ldots & \ldots & \vdots \\
\ldots & \beta_{i} \sigma, & \left(1-\alpha_{i}-\beta_{i}\right) \sigma, & \alpha_{i} \sigma, & \ldots \\
\vdots & \ldots & \ldots & \ddots & \vdots \\
\alpha_{n} \sigma, & 0, & \ldots & \beta_{n} \sigma, & \left(1-\alpha_{n}-\beta_{n}\right) \sigma
\end{array}\right] .
$$

Local stability of the generic fixed point $\overline{\mathbf{X}}$ requires that the largest modulus of eigenvalues evaluated from the characteristic equation $\left|\lambda \mathbf{I}_{n}-\mathscr{F}(\overline{\mathbf{X}})\right|=0$ be less than unity. However, it can be seen that $\sigma$ is also an eigenvalue of $\mathscr{F}$. Therefore, if $\bar{x}$ is an unstable fixed point of $G$, then $\overline{\mathbf{X}}$ must be an unstable fixed point of $\mathbf{F}$ as well.

On the other hand, a generic fixed point is stable if $\sum_{j=1}^{n}\left|J_{i j}\right|<1$, that is,

$$
\left|\left(1-\alpha_{i}-\beta_{i}\right) \sigma\right|+\left|\alpha_{i} \sigma\right|+\left|\beta_{i} \sigma\right|<1
$$

for $i=1,2, \ldots, n$. When $\epsilon_{i}=\alpha_{i}+\beta_{i} \leq 1$ holds for all $i$, the left-hand side of (3.3) simplifies to $|\sigma|$. Therefore, $|\sigma|<1$ would guarantee the stability of $\overline{\mathbf{X}}$.

Nevertheless, it deserves to emphasize that even when a generic fixed point $\overline{\mathbf{X}}$ is locally stable, the convergence of the system to it may not be guaranteed due to the possibility of coexistence of many more stable fixed points and periodic orbits. This phenomenon is particularly true when the system size $n$ is large.

Theorem 2.3 ensures that if the generic fixed point $\overline{\mathbf{X}}$ of (3.1) is either of type I or type II, an implementation of uniformly adaptive adjustment (2.4) should make the fixed point become local stable (local stability is emphasized in this paper instead of the convergency issue as addressed in $[3,4]$ for a noncoupled dynamical system. This is because many unstable fixed points and periodic orbits coexisting in a coupled map lattice system (especially when the system size $n$ is large) may become local stable if an adaptive adjustment mechanism is implemented. Therefore, whether an adjusted system converges to a generic fixed point or other periodic orbits depends on the initial states of the system).

Despite the fact that an unstable fixed point of the one-dimensional map $G(x)$ can be either of type I or type II, an internally coupled lattice system defined by (3.1), however, does have the possibility of possessing a type III fixed point. Such undesired situations can be illustrated with a simple example.

Example 3.5. Consider a four-dimensional uniformly forward-coupled lattice system in the sense that $\alpha_{i}=\alpha$ and $\beta_{i}=0$, for all $i$. Let $\sigma=G^{\prime}(\bar{x})$. Then the Jacobian matrix evaluated 


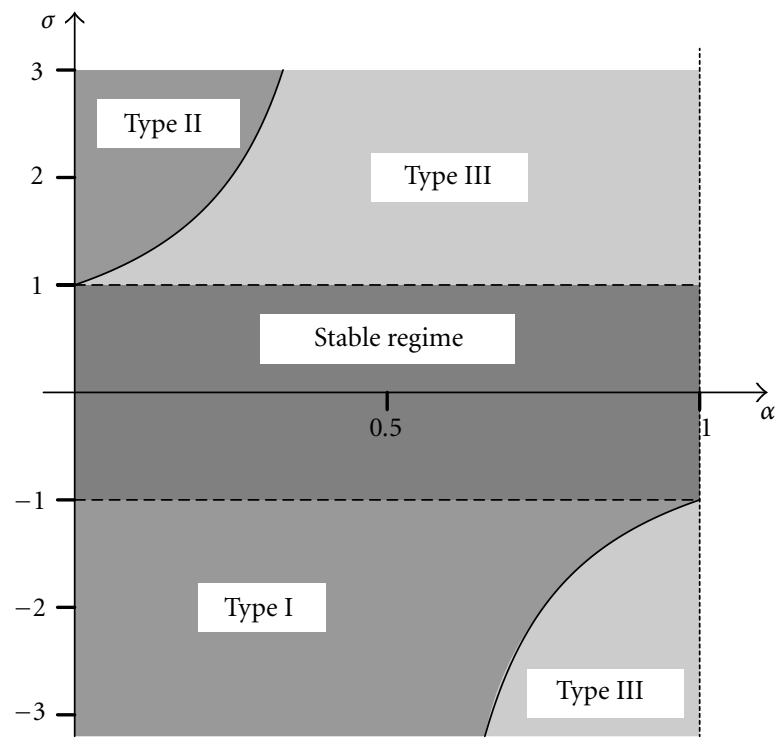

Figure 3.1. Distribution of the generic steady state.

at a fixed point given by

$$
\mathscr{J}=\left[\begin{array}{cccc}
(1-\alpha) \sigma & \alpha \sigma & 0 & 0 \\
0 & (1-\alpha) \sigma & \alpha \sigma & 0 \\
0 & 0 & (1-\alpha) \sigma & \alpha \sigma \\
\alpha \sigma & 0 & 0 & (1-\alpha) \sigma
\end{array}\right]
$$

gives rise to four distinct eigenvalues: $\lambda_{1}=\sigma, \lambda_{2}=(1-2 \alpha) \sigma$, and $\lambda_{3,4}=(1-\alpha) \sigma \pm \mathbf{i} \alpha \sigma$.

If $\sigma<-1$, that is, the fixed point $\bar{x}$ is of type I for the simple one-dimensional map $G$, the internally coupled map lattice has at least one characteristic root that is less than unity $\left(\lambda_{1}=\sigma<-1\right)$. However, $\lambda_{2}=(1-2 \alpha) \sigma$ will be greater than unity if

$$
\alpha>\alpha^{*}(\sigma)=\frac{\sigma-1}{2 \sigma},
$$

which makes the generic fixed point $\mathbf{X}^{-}=(\bar{x}, \bar{x}, \bar{x}, \bar{x})$ a type III one.

Similarly, if $\sigma>1$, that is, the fixed point $\bar{x}$ is of type II for the simple one-dimensional map $G$, then $\overline{\mathbf{X}}$ will be a type III unstable fixed point if $\alpha>\alpha^{*}(\sigma)$.

Figure 3.1 illustrates the distributions of types of unstable fixed points.

Thus, it is reasonable to expect that the stabilization of a strongly internally coupled map lattice system requires nonuniformly adaptive adjustment, the success of which sometimes relies on trial and error. Fortunately, in most practical situations, the map lattice systems are relatively weakly coupled so that a simple uniformly adaptive adjustment can be implemented. However, in the real applications where the system size $n$ is large, it is either unrealistic or impossible to verify whether a fixed point of a coupled lattice 
system is of type III or not. Such a consideration leads us to the next section, where some sufficient conditions will be established so as to provide reliable criteria for practical application.

\section{Uniformly adaptive adjustment in internally coupled map lattice systems}

We start with an implementation of uniformly adaptive adjustment:

$$
\mathbf{X}_{t+1}=\mathbf{F}_{\gamma}=(1-\gamma) \mathbf{F}\left(\mathbf{X}_{t}\right)+\gamma \mathbf{X}_{t}
$$

where $\gamma>0$ and $\mathbf{F}\left(\mathbf{X}_{t}\right)$ is an internally coupled map lattice defined in Definition 3.1. If $\overline{\mathbf{X}}$ is a generic fixed point of $\mathbf{F}$, then $\overline{\mathbf{X}}$ is also a generic fixed point of $\mathbf{F}_{\gamma}$.

With the same notations utilized in the last section and letting

$$
\epsilon_{i}=\alpha_{i}+\beta_{i}<1, \quad \text { for } i=1,2, \ldots, n \text {, }
$$

we arrive at the following conclusions.

Theorem 4.1. (i) If the inequality $1-\left(1-\epsilon_{i}\right) \sigma>\epsilon_{i}|\sigma|$ holds for all $i=1,2, \ldots, n$, there always exists a $\Gamma^{-}=\left(\gamma^{-}, 1\right]$ such that the local stability of the generic fixed point of (4.1) can be guaranteed for $\gamma \in \Gamma^{-}$under uniformly adaptive adjustment, where

$$
\gamma^{-}=1-\min _{i}\left\{\frac{2}{1-\sigma+(\sigma+|\sigma|) \epsilon_{i}}\right\} \text {. }
$$

(ii) If the inequality $\left(1-\epsilon_{i}\right) \sigma-1>\epsilon_{i}|\sigma|$ holds for all $i=1,2, \ldots, n$, there always exists a $\Gamma^{+}=\left[1, \gamma^{+}\right)$such that the local stability of the generic fixed point of (4.1) can be guaranteed for $\gamma \in \Gamma^{+}$under uniformly adaptive adjustment, where

$$
\gamma^{+}=1+\min _{i}\left\{\frac{2}{\sigma+\epsilon_{i}|\sigma|-\sigma \epsilon_{i}-1}\right\} .
$$

Proof. With an implementation of uniformly adaptive adjustment defined by (4.1), the Jacobian matrix of $\tilde{\mathbf{F}}$, evaluated at $\overline{\mathbf{X}}$ and denoted by $\tilde{J}=\left[\tilde{\jmath}_{i j}\right]_{n \times n}$, is thus given by

$$
\tilde{J}=\left[\begin{array}{ccccc}
J_{11} & (1-\gamma) \alpha_{1} \sigma & 0 & \cdots & (1-\gamma) \beta_{1} \sigma \\
\vdots & \ddots & \cdots & \cdots & \vdots \\
\cdots & (1-\gamma) \alpha_{i} \sigma & J_{i i} & (1-\gamma) \beta_{i} \sigma & \cdots \\
\vdots & \ldots & \cdots & \ddots & \vdots \\
(1-\gamma) \beta_{n} \sigma & 0 & \cdots & (1-\gamma) \alpha_{n} \sigma & J_{n n}
\end{array}\right]
$$

where $J_{i i}=(1-\gamma)\left(1-\alpha_{i}-\beta_{i}\right) \sigma+\gamma$.

The stability of $\overline{\mathbf{X}}$ can be guaranteed if $\sum_{j=1}^{n}\left|\tilde{j}_{i j}\right|<1$ holds for all $i$, that is,

$$
\left|(1-\gamma)\left(1-\alpha_{i}-\beta_{i}\right) \sigma+\gamma\right|+\left|(1-\gamma) \alpha_{i} \sigma\right|+\left|(1-\gamma) \beta_{i} \sigma\right|<1
$$

for $i=1,2, \ldots, n$. 


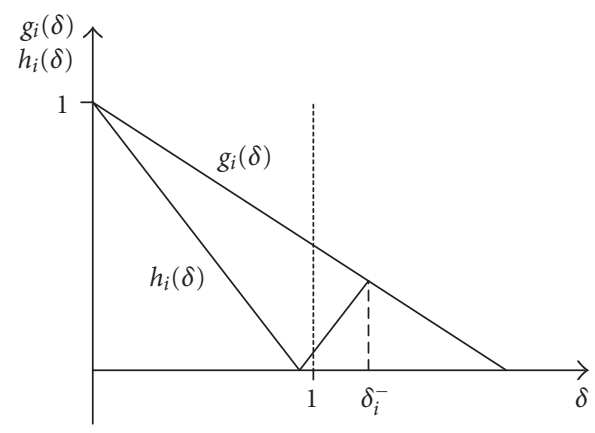

(a)

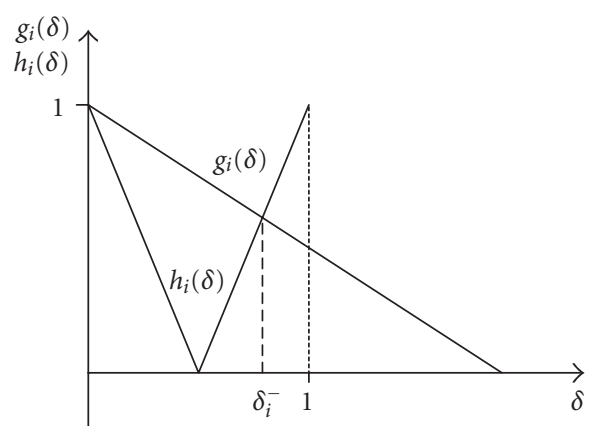

(b)

Figure 4.1. Illustration of existence of $\Delta_{i}^{-}$: (a) $\delta_{i}^{-}>1$ and $\Delta_{i}^{-}=[0,1]$, (b) $\delta_{i}^{-}<1$ and $\Delta_{i}^{-}=\left[0, \delta_{i}^{-}\right]$.

At first, consider the case in which the adjustment is restricted to the conventional range, that is, $0 \leq \gamma<1$. We can define $\gamma=1-\delta$, with $1 \geq \delta \geq 0$.

Let

$$
\begin{aligned}
& g_{i}(\delta)=1-\epsilon_{i} \delta|\sigma|, \\
& h_{i}(\delta)=\left|1-\delta\left[1-\left(1-\epsilon_{i}\right) \sigma\right]\right|,
\end{aligned}
$$

where $\epsilon_{i}$ is defined by (4.2).

Condition (4.6) holds for a particular $i$ if there exists a segment $\Delta_{i}^{-}=\left(0, \delta_{i}^{-}\right) \subset[0,1]$ such that $g_{i}(\delta)>h_{i}(\delta)$ for $\delta \in \Delta_{i}^{-}$. As illustrated in Figure 4.1, this is only possible when

$$
\begin{gathered}
\left(1-\epsilon_{i}\right) \sigma<1, \\
1-\left(1-\epsilon_{i}\right) \sigma>\epsilon_{i}|\sigma| .
\end{gathered}
$$

However, due to the positiveness of $\epsilon_{i}$, inequality (4.8) is also met whenever (4.9) is met.

Let $\delta_{i}^{-}$be the solution of $g_{i}\left(\delta_{i}^{-}\right)=h_{i}\left(\delta_{i}^{-}\right)$, that is,

$$
1-\epsilon_{i}|\sigma| \delta=\delta\left[1-\left(1-\epsilon_{i}\right) \sigma\right]-1,
$$

which yields

$$
\delta_{i}^{-}=\frac{2}{1-\sigma+(\sigma+|\sigma|) \epsilon_{i}}
$$

Next, we examine the generalized adjustment range, that is, $\gamma>1$. We can similarly define $\gamma=1+\delta$, with $\delta \geq 0$.

With $g_{i}(\delta)=1-\epsilon_{i} \delta|\sigma|$, we redefine

$$
h_{i}(\delta)=\left|1-\delta\left[\left(1-\epsilon_{i}\right) \sigma-1\right]\right| .
$$


By a similar reasoning, condition (4.6) holds for a particular $i$ if there exists a segment $\Delta_{i}^{+}=\left(1, \delta_{i}^{+}\right) \subset[1, \infty)$ such that $g_{i}(\delta)>h_{i}(\delta)$ for $\delta \in \Delta_{i}^{+}$, which is only possible when

$$
\left(1-\epsilon_{i}\right) \sigma-1>\epsilon_{i}|\sigma|
$$

Let $\delta_{i}^{+}$be the solution of $g_{i}\left(\delta_{i}^{+}\right)=h_{i}\left(\delta_{i}^{+}\right)$. Then it can be verified that

$$
\delta_{i}^{+}=\frac{2}{\sigma+\epsilon_{i}|\sigma|-\sigma \epsilon_{i}-1} .
$$

We also notice that the restriction that $\delta_{i}^{+}>0$ is guaranteed when condition (4.13) is satisfied.

Denote $\gamma_{i}^{-}=1-\delta_{i}^{-}$and $\gamma_{i}^{+}=1+\delta_{i}^{+}$. So long as either one of conditions (4.9) and (4.13) is met, the local stability of the generic fixed point can be easily guaranteed with $\gamma>\max _{i} \gamma_{i}^{-}$or $\gamma<\min _{i} \gamma_{i}^{+}$, respectively.

\section{Uniformly internally coupled map lattice systems}

For a uniformly internally coupled map lattice with $\epsilon_{i}=\epsilon$ for all $i$, Theorem 4.1 can be simplified to the following theorem.

THEOREM 5.1. For a uniformly internally coupled map lattice, a set of sufficient conditions for the local stability of a generic fixed point $\overline{\mathbf{X}}$ with the implementation of uniformly adaptive adjustment is as follows:

(i) $\epsilon<\epsilon^{*}=(1 / 2)((\sigma-1) / \sigma)$,

(ii) $\gamma>\gamma^{*}=(\sigma+1) /(\sigma-1)$.

Remark 5.2. (i) The set of sufficient conditions stated in Theorem 5.1 is independent of the size $n$ of the coupled map lattice. Moreover, the conditions depend only on the sum of $\alpha$ and $\beta$ instead of their individual values.

(ii) As long as the condition $\epsilon<\epsilon^{*}$ holds, which is generally true for weak coupling, the range of adaptive adjustment parameter that guarantees the local stability is independent of $\epsilon$.

(iii) In particular, as depicted in Figure 5.1, stabilization regimes can be classified on a $(\sigma, \epsilon)$-plane with

(a) original stable regime $\mathscr{S}$ formed by $|\sigma|<1$,

(b) regime $\mathscr{S}^{+}$formed by $\sigma>1$ and $\epsilon<\epsilon^{*}$, in which the stability can be guaranteed for $\gamma^{*}>\gamma>1$,

(c) regime $\mathscr{S}^{-}$formed by $\sigma<-1$ and $\epsilon<\epsilon^{*}$, in which the stability can be guaranteed for $1>\gamma>\gamma^{*}$.

The above remarks can be made clearer by noticing the similarity between Figures 3.1 and 5.1. 


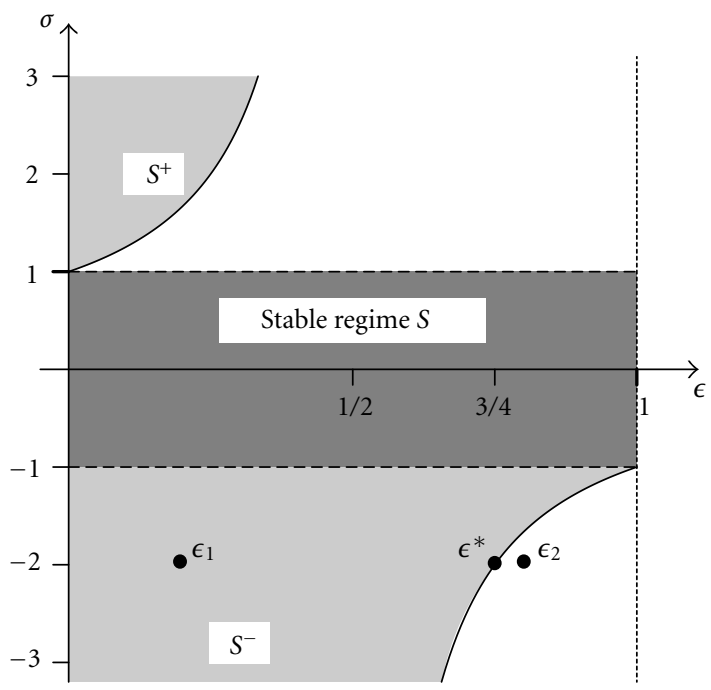

Figure 5.1. Stability regimes for $x_{i, t+1}=G\left((1-\epsilon) x_{i, t}+\alpha x_{i+1, t}+\beta x_{i-1, t}\right)$, where $\epsilon=\alpha+\beta$.

\section{Numerical simulations}

Example 6.1 (internally coupled logistic system). Consider a case of uniformly internally coupled map lattice system with the most studied logistic equation

$$
G(x)=4 x(1-x)
$$

as a coupling map. We simulate a coupled map lattice with a system size $n=100$ and $\alpha=\beta=\epsilon / 2$;

$$
\begin{aligned}
x_{i, t+1}= & f_{i}\left(\mathbf{X}_{\mathbf{t}}\right)=G\left((1-\epsilon) x_{i, t}+\frac{\epsilon}{2} x_{i+1, t}+\frac{\epsilon}{2} x_{i-1, t}\right) \\
= & 4\left((1-\epsilon) x_{i, t}+\frac{\epsilon}{2} x_{i+1, t}+\frac{\epsilon}{2} x_{i-1, t}\right)\left(1-x_{i, t}+\epsilon x_{i, t}-\frac{\epsilon}{2} x_{i+1, t}-\frac{\epsilon}{2} x_{i-1, t}\right) \\
= & \left(2(1-\epsilon) x_{i, t}+\epsilon\left(x_{i+1, t}+x_{i-1, t}\right)\right)\left(2\left(1-x_{i, t}\right)-\epsilon\left(-2 x_{i, t}+x_{i+1, t}+x_{i-1, t}\right)\right) \\
= & 4(1-\epsilon) x_{i, t}\left(1-x_{i, t}\right)+2 \epsilon\left(1-x_{i, t}\right)\left(x_{i+1, t}+x_{i-1, t}\right) \\
& -2 \epsilon(1-\epsilon) x_{i, t}\left(-2 x_{i, t}+x_{i+1, t}+x_{i-1, t}\right)-\epsilon^{2}\left(x_{i+1, t}+x_{i-1, t}\right)\left(-2 x_{i, t}+x_{i+1, t}+x_{i-1, t}\right),
\end{aligned}
$$

$G(x)$ has a unique nontrivial fixed point $\bar{x}=3 / 4$, at which the derivative is given by $\sigma=$ $G^{\prime}(\bar{x})=-2$. 


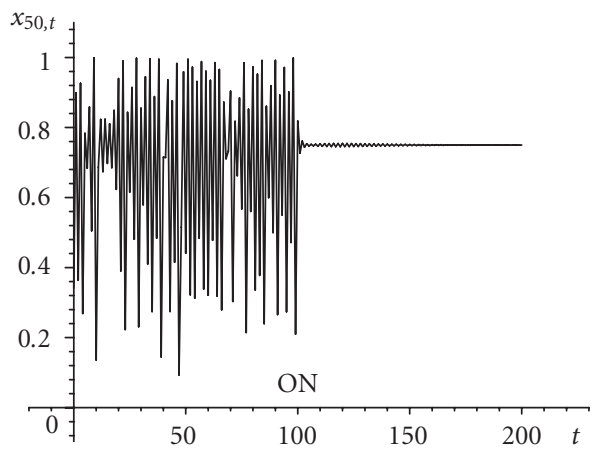

(a)

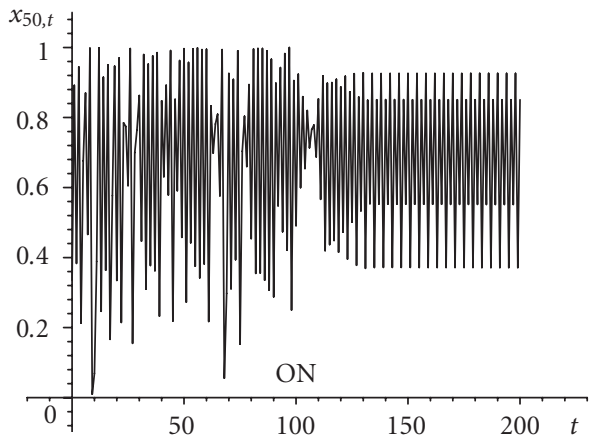

(b)

Figure 6.1. Simulations for a weakly coupled map lattice system $\left(\epsilon^{1}=1 / 5 \ll \epsilon^{*}\right)$ : (a) $\epsilon=1 / 5, \gamma=$ 0.35 , (b) $\epsilon=1 / 5, \gamma=0.15$. The adaptive adjustment is trigged at 101th step. Initial states are given randomly around 0.3333 .

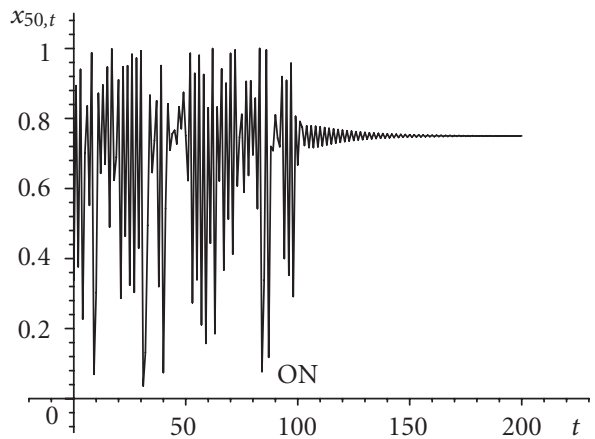

(a)

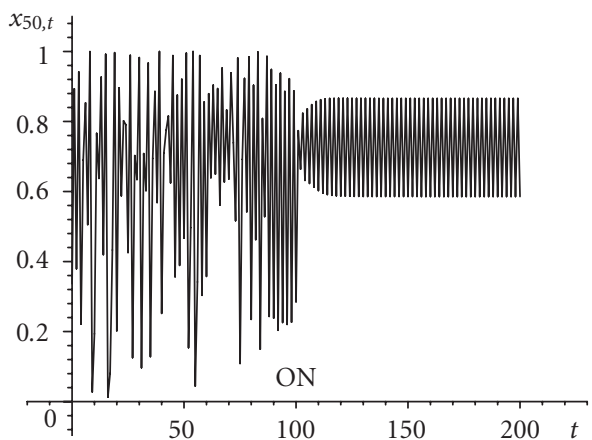

(b)

Figure 6.2. Simulations for a coupled map lattice system at critical coupling value $\epsilon^{*}$ : (a) $\epsilon=3 / 4$, $\gamma=0.35$, (b) $\epsilon=3 / 4, \gamma=0.15$. The adaptive adjustment is trigged at 101th step. Initial states are given randomly around 0.3333 .

By referring to Figure 5.1, it can be seen that when $\epsilon<\epsilon^{*}=3 / 4$, a uniformly adaptive adjustment with a parameter range $\gamma \in\left[\gamma^{*}, 1\right]$ would stabilize the system to the generic fixed point given by $\overline{\mathbf{X}}=(3 / 4,3 / 4, \ldots, 3 / 4)$, where $\gamma^{*}=(-2+1) /(-2-1)=1 / 3$.

Computer simulations for several $(\epsilon, \gamma)$ combinations are presented in Figures 6.1, 6.2, and 6.3, where the first 100 iterations are carried out without adaptive adjustments and the adjustments are implemented after the 100th step. 


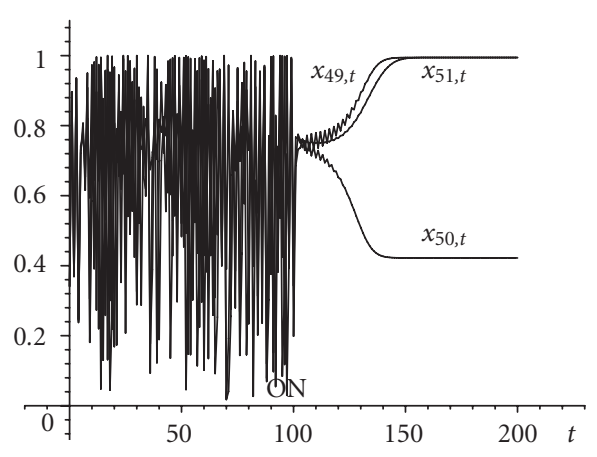

(a)

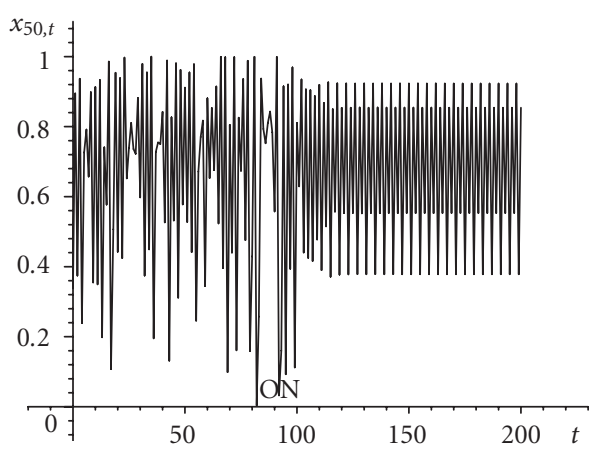

(b)

Figure 6.3. Simulations for a strongly coupled map lattice system $\left(\epsilon^{2}=4 / 5>\epsilon^{*}\right)$ : (a) $\epsilon=4 / 5, \gamma=$ 0.35 , (b) $\epsilon=4 / 5, \gamma=0.15$. The adaptive adjustment is trigged at 101th step. Initial states are given randomly around 0.3333 .

The case depicted in Figure 6.1 is assumed with a relatively weak coupling $\left(\epsilon_{1}=1 / 4<\right.$ $\left.\epsilon^{*}\right)$. When $\gamma=0.35$, the system converges to the generic fixed point just in a few iterations. When $\gamma=0.15$, significantly smaller than the lower bound $\gamma^{*}$, the trajectory converges to a stable periodic cycle instead.

Figure 6.2 depicts the case in which the system is coupled at the critical boundary of stabilization regime: $\epsilon=3 / 4=\epsilon^{*}$. Nevertheless, the convergence to the generic fixed point is still achieved for $\gamma=0.35$. Again, the system converges to a stable periodic orbit for $\gamma=1 / 4$.

To demonstrate the effectiveness and efficiency, we present a case of a very strong coupling $\left(\epsilon_{2}=4 / 5>\epsilon^{*}\right)$. Even though this case is beyond the stability regimes guaranteed in Figure 5.1, an implementation of uniformly adaptive adjustment with $\gamma=0.35$ still successfully forces the system to converge to a fixed point. The fixed point converged, however, is not generic. Figure 6.3(a) plots three nearby trajectories: $x_{49, t}, x_{50, t}$, and $x_{51, t}$. Again, when $\gamma=0.15$, a typical trajectory converges to a periodic cycle, which is depicted in Figure 6.3(b) (this periodic orbit may not be generic in the sense that it is also a periodic orbit of map $G$. However, the same technique can be applied to stabilize an internally coupled map lattice to a periodic orbit. Due to the limited scope of this paper, the simulations are omitted upon the request of the editor).

\section{Conclusion}

We have proved in a theory that a uniformly adaptive adjustment can be utilized to stabilize an internally coupled map lattice system to its generic fixed point and/or periodic orbits. Simulations conducted by us have shown that such stabilization turns out to be very effective and efficient. Convergence is usually achieved soon after adaptive adjustment is triggered. 


\section{Stabilization of internally coupled map lattice}

\section{References}

[1] R. O. Grigoriev and M. C. Cross, Controlling physical systems with symmetries, Phys. Rev. E 57 (1998), 1550-1554.

[2] G. Hu and Z. Qu, Controlling spatiotemporal chaos in coupled map lattice systems, Phys. Rev. Lett. 72 (1994), 68-71.

[3] W. Huang, Stabilizing unstable discrete systems by a nonuniformly adaptive adjustment mechanism, Phys. Rev. E 62 (2000), no. 3, 3455-3460.

[4] , Theory of adaptive adjustment, Discrete Dyn. Nat. Soc. 5 (2001), no. 4, 247-263.

[5] _ Stabilizing coupled map lattice systems with adaptive adjustment, Phys. Rev. E 66 (2002), no. 3, 1-7.

[6] N. Parekh, S. Parthasarathy, and S. Sinha, Global and local control of spatiotemporal chaos in coupled map lattices, Phys. Rev. Lett. 81 (1998), 1401-1404.

[7] P. Parmananda, R. Madrigal, M. Rivera, L. Nyikos, I. Z. Kiss, and V. Gáspár, Stabilization of unstable steady states and periodic orbits in an electrochemical system using delayed-feedback control, Phys. Rev. E 59 (1999), no. 5, 5266-5271.

[8] S. Sinha and N. Gupte, Adaptive control of spatially extended systems: targeting spatiotemporal patterns and chaos, Phys. Rev. E 58 (1998), no. 5, R5221-R5224.

[9] Z. Xu and K. Shen, Controlling spatiotemporal chaos via phase space compression, Phys. Rev. E 63 (2001), no. 4, 1-6.

Weihong Huang: Division of Economics, School of Humanities and Social Sciences, Nanyang Technological University, Nanyang Avenue, Singapore 639798

E-mail address: awhhuang@ntu.edu.sg 


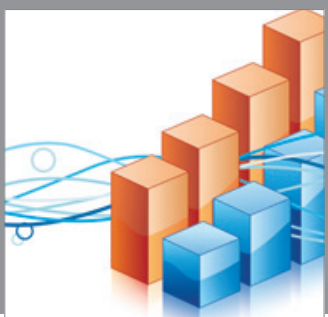

Advances in

Operations Research

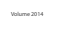

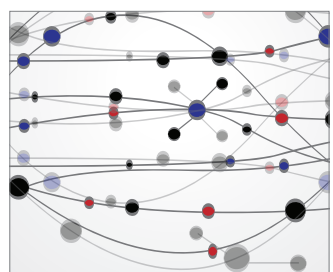

\section{The Scientific} World Journal
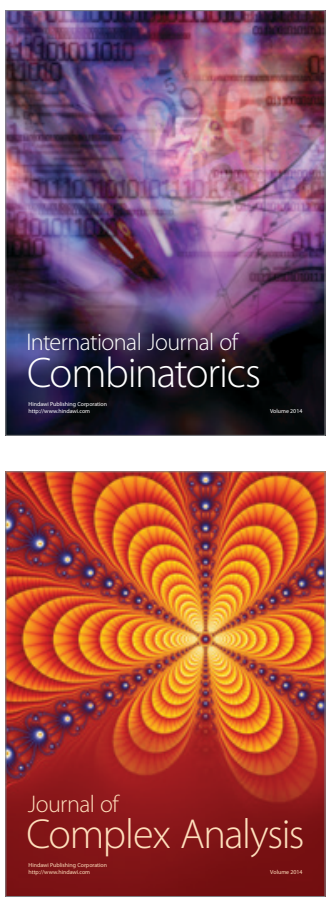

International Journal of

Mathematics and

Mathematical

Sciences
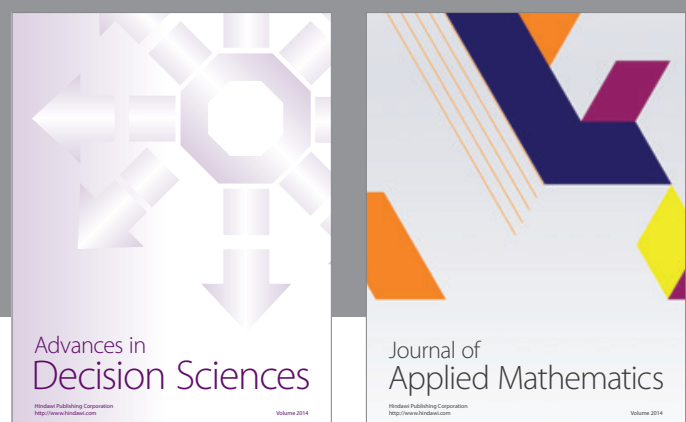

Journal of

Applied Mathematics
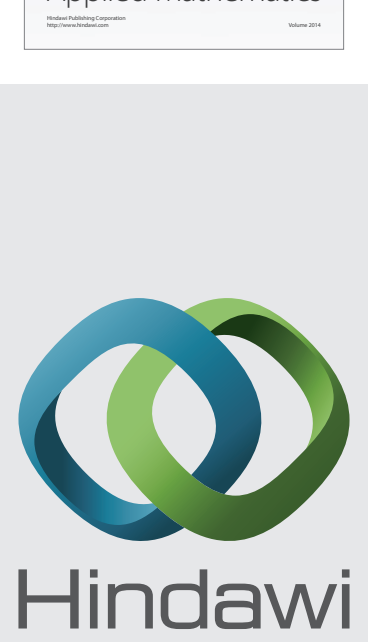

Submit your manuscripts at http://www.hindawi.com
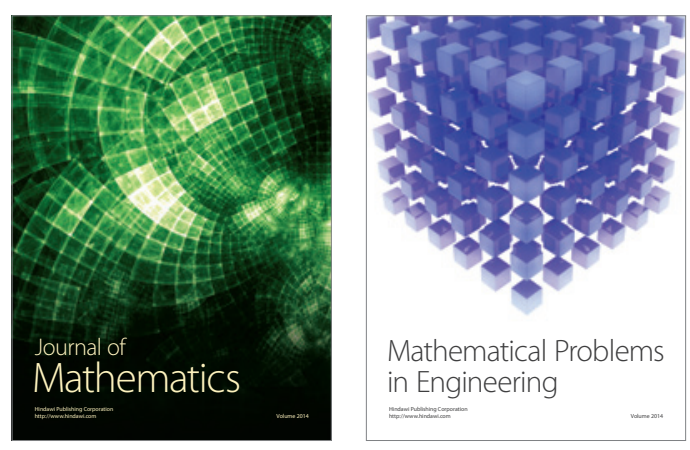

Mathematical Problems in Engineering
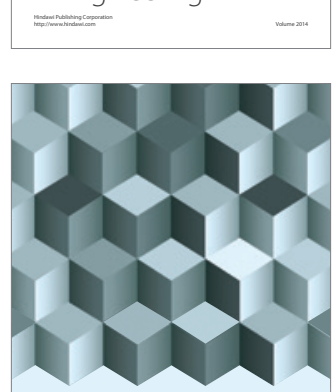

Journal of

Function Spaces
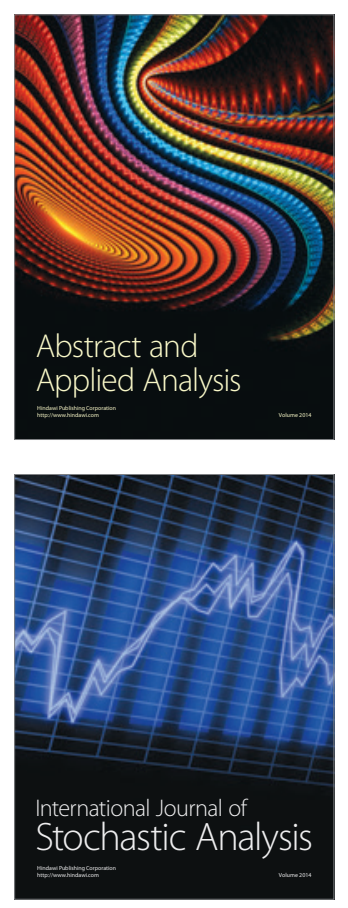

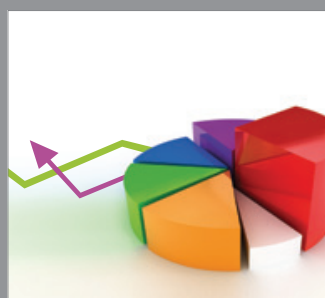

ournal of

Probability and Statistics

Promensencen
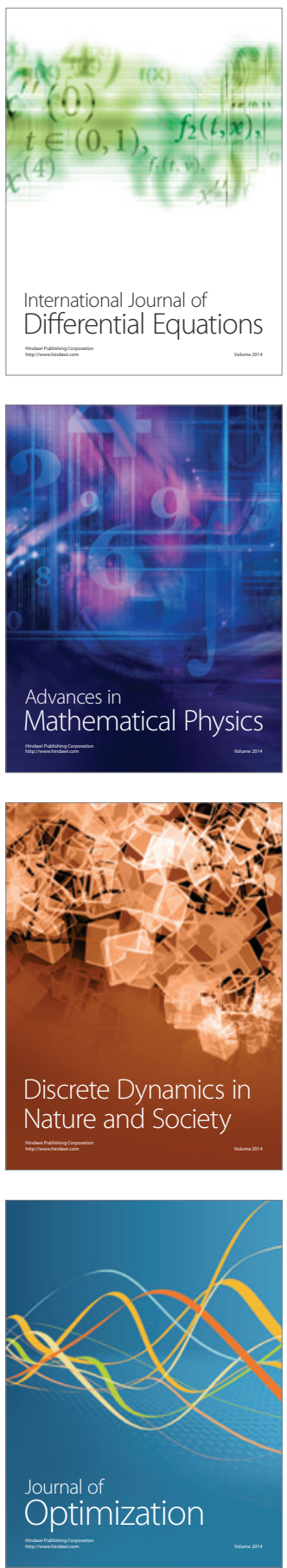